\title{
Distant lung metastases caused by a histologically benign phosphaturic mesenchymal tumor
}

\author{
Maria P Yavropoulou', Christos Poulios², Christoforos Foroulis³, Symeon Tournis4, Prodromos Hytiroglou², \\ Kalliopi Kotsa', Isaak Kessisoglou ${ }^{5}$ and Pantelis Zebekakis
}

1Division of Endocrinology and Metabolism, 1st Department of Internal Medicine, AHEPA University Hospital, Thessaloniki, Greece, 2Department of Pathology, Faculty of Medicine, Aristotle University of Thessaloniki, Greece, 3Department of Thoracic Surgery, AHEPA University Hospital, Thessaloniki, Greece, 4Laboratory of Research of Musculoskeletal System 'Th. Garofalidis', KAT Hospital University of Athens, Greece, and 53rd Department of Surgery, AHEPA University Hospital, Thessaloniki, Greece

Correspondence should be addressed to M P Yavropoulou Email

margia@med.auth.gr

\section{Summary}

Tumor-induced osteomalacia (TIO) is a rare form of hypophosphatemia usually caused by phosphaturic mesenchymal tumors (PMTs); the biologic behavior of PMTs is under investigation. Herein we present a case of TIO with a protracted course over 12 years leading to a fatal outcome. A 39-year-old man presented with weakness in 2004 and was found to have decreased serum phosphorus, phosphaturia and low levels of 1,25-dihydroxyvitamin D3. Four years later he developed a painful left calf mass. The lesion was resected, but recurred causing extreme pain and dysfunction. Radiological examination showed a large cluster of soft tissue tumors affecting all the muscle compartments of the calf and a smaller lesion inside the metaphysis of the tibia. Above-knee amputation was performed. Histological examination of all lesions showed a cellular spindle cell neoplasm with variously sized vessels, wide vessel-like spaces and scattered deposits of calcified extracellular material. The tumor infiltrated skeletal muscles, subcutaneous fat and the proximal end of the fibula. The tibial lesion had identical histology. Three years after the amputation the patient presented with cough and dyspnea. Radiological examination, followed by an open biopsy, showed that there were multiple metastatic nodules of PMTs in both lungs. Shortly after the diagnosis the patient died. This case illustrates that even benign cases of PMTs may lead to a fatal outcome and the classification of PMTs into benign and malignant should be reassessed in order to correspond to its biological behavior.

\section{Learning points:}

- PMTs, aside from having locally aggressive behavior, may metastasize and cause death

- PMTs may behave aggressively despite 'benign' histological findings

- Accurate diagnosis of tumor-induced osteomalacia and patient management require a multidisciplinary approach

\section{Background}

Tumor-induced osteomalacia (TIO) is a rare form of hypophosphatemia caused by phosphaturic mesenchymal tumors (PMTs) located in the bone or soft tissue. The diagnosis is usually based on the biochemical profile of severely low levels of phosphate $\left(\mathrm{PO}_{4}\right)$ associated with phosphaturia and low or inappropriately normal levels of 1,25-OH-vitamin D. Increased levels of fibroblast growth factor 23 (FGF 23) that is excreted by the tumor is the 
pathophysiological factor responsible for phosphaturia. Localization and resection of the tumor can cure completely osteomalacia. The majority of PMTs are benign, and malignancy or malignant transformation of PMTs with distant metastasis is very uncommon (1). We describe herein the follow-up of a previously reported case (2) with a histopathologicaly benign phosphaturic mesenchymal tumor that caused severe hypophosphatemia and multiple recurrences, and finally led to amputation of the affected limb. Although there was no evidence of histologic malignancy, 13 years after the initial diagnosis and 2 years after amputation the patient developed fatal lung metastases.

\section{Case presentation}

This is a follow-up presentation of a case that was part of our study registered on Clinical trials.gov (protocol number NCT01660308) and approved by the Scientific Review Board of AHEPA University Hospital (protocol number 51741).

Our patient presented with a medical history of TIO caused by a histopathologically benign PMTs with a locally aggressive behavior, which led to multiple recurrences (2).

On his first admission to our center the patient had severe hypophosphatemia (1.8 mg/dL, RR: $2.7-4.5 \mathrm{mg} / \mathrm{dL})$ and elevated $24 \mathrm{~h}$ urine phosphate $(1797.4 \mathrm{mg} / 24 \mathrm{~h}$, RR: $400-1300 \mathrm{mg} / 24 \mathrm{~h}$ ), elevated alkaline phosphatase (ALP) levels (300IU/L, RR: 40-150IU/L), elevated parathyroid hormone (PTH) (19.4 pmol/L, RR: $1.8-6.03 \mathrm{pmol} / \mathrm{L})$, and serum $\mathrm{Ca}^{2+}$ levels $(10.8 \mathrm{mg} / \mathrm{dL}$, RR: $8.2-10.6 \mathrm{mg} / \mathrm{dL})$, and low to normal 1,25 (OH) ${ }_{2}$-vitamin D levels $(18 \mathrm{pg} /$ $\mathrm{mL}, \mathrm{RR}: 18-24 \mathrm{pg} / \mathrm{mL})$ and 25-OH-vitamin D (20.9 ng/ $\mathrm{mL}$, RR: $40-100 \mathrm{ng} / \mathrm{mL}$ ), as previously described (2). The ratio of maximum rate of renal tubular reabsorption of phosphate to glomerular filtration rate $\left(\mathrm{TmPO}_{4} / \mathrm{GFR}\right)$ as determined by the Walton and Bijvoet nomogram was noted to be low at $0.3 \mathrm{mmol} / \mathrm{L}(0.8-1.4)$ confirming the excessive loss of phosphate in the urine (2). There were no signs of glycosuria, aminoaciduria or proteinuria. In our initial approach of the patient, the medical team decided resection of the tumor mass which was located in the periphery of the head and the upper third of the left fibular diaphysis due to the patient's medical record of repeated recurrences and his biochemical profile of markedly decreased phosphate and $1,25(\mathrm{OH})_{2}$ vitamin D levels and significantly increased plasma FGF-23 levels. Radiological examination of the left limp showed that the mass of the calf consisted of multiple tumors and that there was a separate lesion at the tibial metaphysis (2). The above-mentioned data combined with the clinical presentation of the patient with excruciating pain and limp dysfunction led to the decision of an aggressive operation of high amputation of the affected limb up to the height of the distal femur. At that time, we also performed a molecular analysis of biopsies taken from the tumor and from cells isolated from bone marrow. We recorded an over-expression of genes related to growth factor receptors (PDGFRA, PDGFRB and VEGFR3) that have been documented before in various types of cancers $(3,4)$, but not in histologically benign PMTs.

Two years after amputation, the patient had persistently low $\mathrm{PO}_{4}$ levels $(1.8 \mathrm{mg} / \mathrm{dL})$, despite being supplemented with oral phosphate and calcitriol; FGF23 levels were also markedly increased $(5630 \mathrm{pg} / \mathrm{mL})$. The tubular reabsorption of phosphate (TRP, 75.97\%), TmP/ $\operatorname{GFR}(0.5 / 24 \mathrm{~h})$, and urinary phosphorus $(29.38 \mathrm{mmol} / \mathrm{L})$, were indicative of hyperphosphaturia. Apart from general muscle weakness and cramps, attributed to chronic hypophosphatemia, he complained of persistent cough and shortness of breath that was aggravated in the last 4 months, restricting him from operating simple everyday tasks and deteriorating even more his quality of life.

\section{Investigation}

A chest X-ray revealed multiple pulmonary nodules with ground glass appearance in both lungs and a PET scan was performed to further evaluate the multiple pulmonary nodules (Fig. 1).

Open lung biopsy was performed. Histological examination of the excised lung nodule revealed metastatic PMTs (Fig. 2). The histologic features were similar to those of the primary tumor (2) and there was no mitotic activity in at least 40 high power fields ( $\times 400$ magnification; Fig. 3 ). The cellular proliferation marker, Ki/67/MIB1 was positive in less than $5 \%$ of the neoplastic cells (Fig. 4). The neoplastic cells were positive for vimentin, and negative for desmin S 100 protein and CD34 antigen. The stroma had areas of hyalinization, ossification and hemosiderin deposits.

\section{Outcome and follow-up}

Six months after the diagnosis of lung metastases, the patient was admitted to the emergency department due to acute dyspnea and ultimately succumbed to respiratory failure $24 \mathrm{~h}$ later 


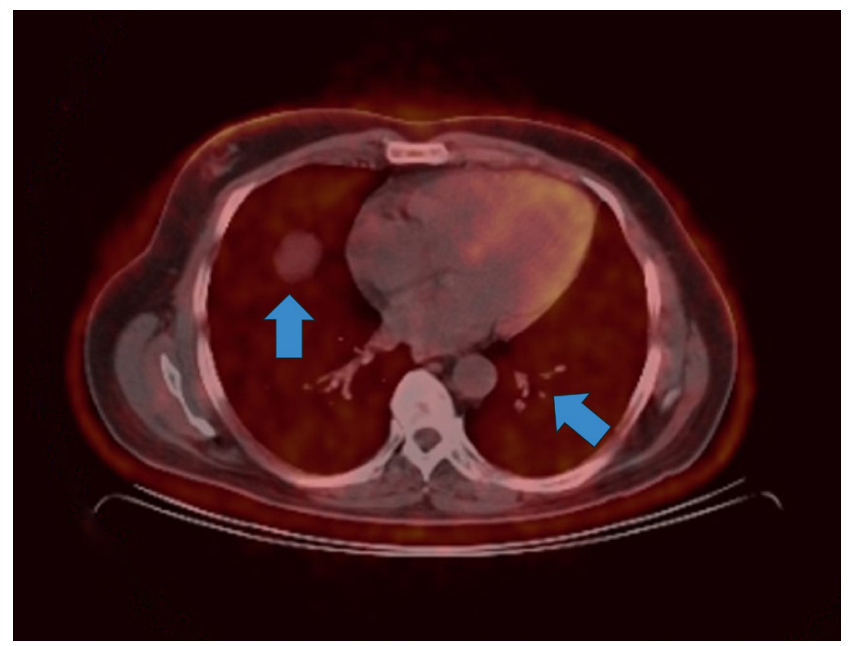

Figure 1

Axial PET-CT scan image showing numerous metabolically active pulmonary nodules.

\section{Discussion}

The histopathological diagnosis of PMTs is a challenge, considering the rarity of the tumor and its nonspecific histological and immunohistochemical findings. As demonstrated by Folpe and et al. (5) most PMTs cases compose of small, bland, spindled cells that produce a distinctive 'smudgy' matrix, with a well-developed capillary network (often described as Hemangiopericytoma-like vessels) and possibly microcystic change. This matrix calcifies in an unusual flocculent or 'grungy' pattern. Mitotic activity is usually mild (0-1 mitoses per high power magnification field). Immunohistochemistry does not positively contribute

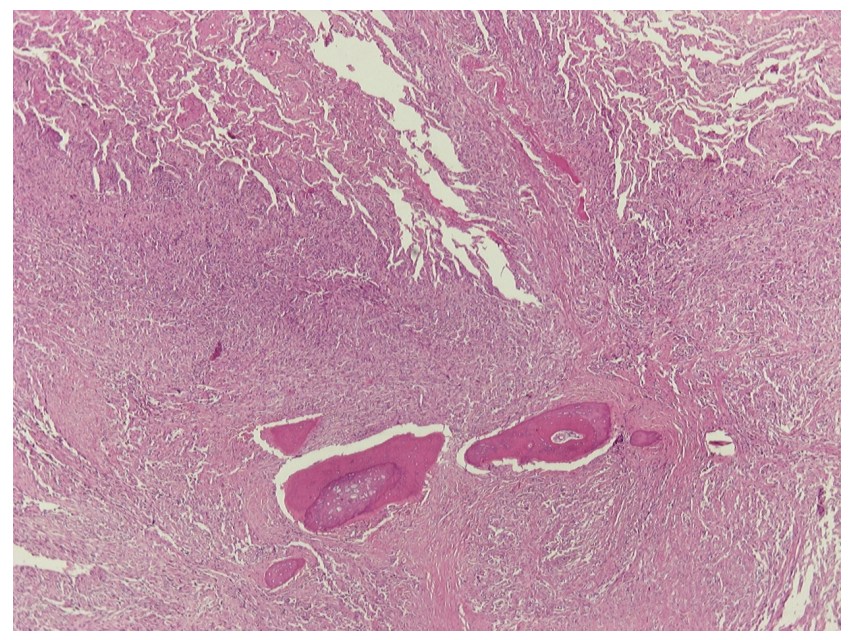

Figure 2

Lung metastasis of PMTs with ossification, H\&E stain $\times 40$.

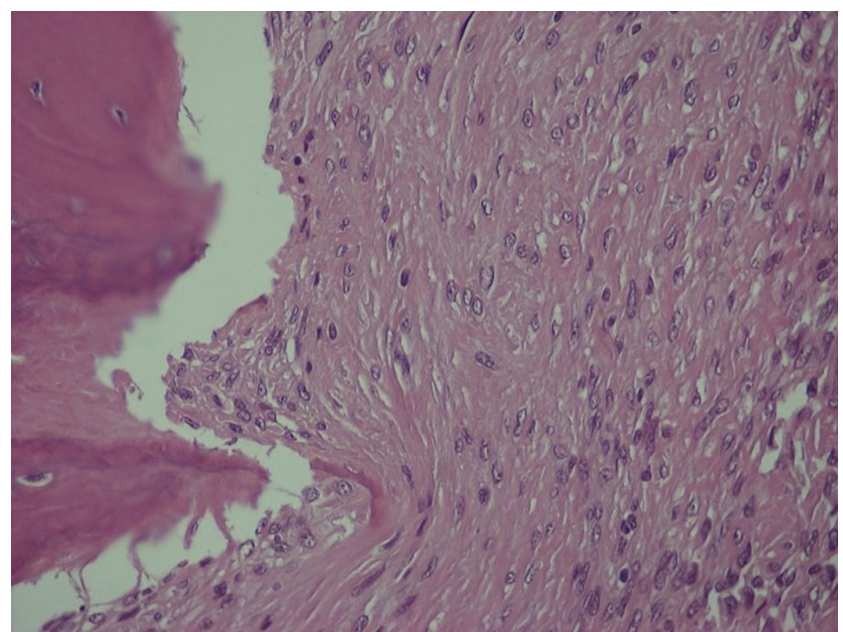

Figure 3

Lung metastasis of PMTs, H\&E stain $\times 400$.

to the diagnosis of PMTs, other than the exclusion of other mesenchymal neoplasms. The exception to this is the immunohistochemical detection of FGF23 expression. Nevertheless, commercially available FGF23 antibodies lack sufficient specificity to reliably diagnose PMTs (6).

The rarity and the elusive histopathological presentation of PMTs have resulted in poor understanding of its biological behavior. According to the World Health Organization (WHO) classification, PMTs has two types: benign and malignant (4). While there are no standard distinctive histopathological criteria between the two forms, the latter is defined on the basis of 'frankly sarcomatous features' (high mitotic rate, prominent atypia and pleomorphism and necrosis). Most reported

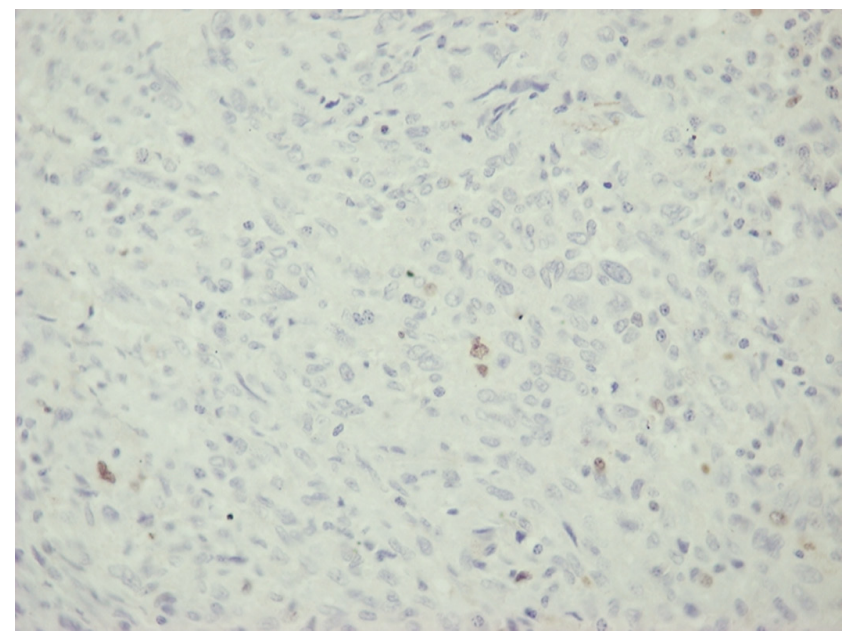

Figure 4

Lung metastasis of PMTs, Ki67/MIB1 stain $\times 400$. 
Table 1 Reported cases of PMTs with malignant behavior.

\begin{tabular}{|c|c|c|c|}
\hline Publication & $\begin{array}{c}\text { Number of } \\
\text { cases }\end{array}$ & Site & $\begin{array}{l}\text { Histopathological evidence } \\
\text { of malignancy }\end{array}$ \\
\hline Folpe et al. (5) & 1 out of 32 & Various & $\begin{array}{l}3 \text { of } 32 \text { cases malignant } \\
\text { PMTs }\end{array}$ \\
\hline
\end{tabular}

Morimoto

et al. (10)

2

(1) Right pelvis (2)
right pelvis (found
at the age of 25
untreated until
the age of 31)

(1) Malignant PMTs (2)

first diagnosis: benign PMTs; metastasis

diagnosis: malignant transformation

No and Ki67 $<3 \%$

(1) Yes (2) yes

nasal cavity

Right leg
Metastasis

Follow-up/outcome

For those patients with available follow-up all were alive free of disease except for four patients with recurrent tumors. One of those developed lung and bone metastasis and was diagnosed with malignant PMTs

(1) Lung and (1) DOD from rapidly progressive bones (2) lung metastases 32 months after lung, bones, second embolization (2) liver and recurrence 2 years after surgery, elbow metastasis, DOD due to respiratory failure (total clinical course: 26 years)

Lung and soft 24 year-course, multiple tissue metastases, AWD

Lung-lung (1) $>20$ year-course multiple recurrences $(2)>10$ year-course multifocal disease

Recurrence after first surgery, AWNED 10 months after the last operation

AWD, alive with disease; AWENED, alive with no evidence of disease; DOD, dead of disease; PMTs, phosphaturic mesenchymal tumors.

cases of PMTs are of the benign type and have either a favourable outcome or recurred locally. Cases of PMTs with distant metastases are rare and usually have evident histological features of malignancy $(5,7,8,9,10)$ (Table 1$)$. To the best of our knowledge the current case is only the second report of PMTs with histologically benign features presenting with distant metastasis on long-term follow-up. In the other case, reported by Qari et al. (8), the patient presented with a $1.2 \mathrm{~cm}$ destructive osteolytic lesion in the mandible and the biopsy showed a cellular spindle cell neoplasm with osteoid-like production, compatible with PMTs. After multiple local recurrences, the tumor eventually metastasized to the lungs as multiple, small foci of tumor. Seventeen years from original presentation, it was confirmed that the disease had become widespread with lesions in the soft tissue. Our case was diagnosed as benign PMTs based on the WHO classification criteria, due to lack of sarcomatous features in all examined specimens, including metastatic sites. The only finding that was suggestive of an aggressive behavior was overexpression of genes related to growth factor receptors (2). Our patient had multiple recurrences, distant metastases and eventually died of the disease, indicating that despite absence of histologic evidence, the tumor had malignant potential.

This case emphasizes the need for long-term follow-up for patients with PMTs.

\section{Declaration of interest}

The authors declare that there is no conflict of interest that could be perceived as prejudicing the impartiality of the research reported.

\section{Funding}

This research did not receive any specific grant from any funding agency in the public, commercial or not-for-profit sector.

\section{Patient consent}

Written informed consent from the patient was obtained before any analysis was performed.

Author contribution statement

M P Yavropoulou wrote the first draft. C Poulios and P Hytiroglou performed the pathology; C Foroulis, S Tournis, I Kesisoglou, K Kotsa, John $\mathrm{G}$ Yovos and $\mathrm{P}$ Zebekakis, revised and approved the final draft.

\section{References}

1 Hautmann AH, Hautmann MG, Kolbl O, Herr W \& Fleck M. Tumorinduced osteomalacia: an up-to-date review. Current Rheumatology Reports 201517 512. (https://doi.org/10.1007/s11926-015-0512-5)

2 Yavropoulou MP, Gerothanasi N, Frydas A, Triantafyllou E, Poulios C, Hytiroglou P, Apostolou P, Papasotiriou I, Tournis S, Kesisoglou I, et al. Tumor-induced osteomalacia due to a recurrent mesenchymal tumor overexpressing several growth factor receptors. Endocrinology, Diabetes and Metabolism Case Reports 20152015 ID:150025. (https:// doi.org/10.1530/EDM-15-0025) 
3 Cebe-Suarez S, Zehnder-Fjallman A \& Ballmer-Hofer K. The role of VEGF receptors in angiogenesis; complex partnerships. Cellular and Molecular Life Sciences 200663 601-615. (https://doi.org/10.1007/ s00018-005-5426-3)

4 Farooqi AA, Waseem S, Riaz AM, Dilawar BA, Mukhtar S, Minhaj S, Waseem MS, Daniel S, Malik BA, Nawaz A, et al. PDGF: the nuts and bolts of signalling toolbox. Tumor Biology 201132 1057-1070. (https://doi.org/10.1007/s13277-011-0212-3)

5 Folpe AL, Fanburg-Smith JC, Billings SD, Bisceglia M, Bertoni F, Cho JY, Econs MJ, Inwards CY, Jan de Beur SM, Mentzel T, et al. Most osteomalacia-associated mesenchymal tumors are a single histopathologic entity: an analysis of 32 cases and a comprehensive review of the literature. American Journal of Surgical Pathology 200428 1-30. (https://doi.org/10.1097/00000478-200401000-00001)

6 Carter JM, Caron BL, Dogan A \& Folpe AL. A novel chromogenic in situ hybridization assay for FGF23 mRNA in phosphaturic mesenchymal tumors. American Journal of Surgical Pathology 201539 75-83. (https://doi.org/10.1097/PAS.0000000000000290)
7 Qiu S, Cao LL, Qiu Y, Yan P, Li ZX, Du J, Sun LM \& Zhang QF. Malignant phosphaturic mesenchymal tumor with pulmonary metastasis: a case report. Medicine 201796 e6750. (https://doi. org/10.1097/MD.0000000000006750)

8 Qari H, Hamao-Sakamoto A, Fuselier C, Cheng YS, Kessler H \& Wright J. Phosphaturic mesenchymal tumor: 2 new oral cases and review of 53 cases in the head and neck. Head and Neck Pathology 201610 192-200. (https://doi.org/10.1007/s12105015-0668-3)

9 Wasserman JK, Purgina B, Lai CK, Gravel D, Mahaffey A, Bell D \& Chiosea SI. Phosphaturic mesenchymal tumor involving the head and neck: a report of five cases with FGFR1 fluorescence in situ hybridization analysis. Head and Neck Pathology 201610 279-285. (https://doi.org/10.1007/s12105-015-0678-1)

10 Morimoto T, Takenaka S, Hashimoto N, Araki N, Myoui A \& Yoshikawa H. Malignant phosphaturic mesenchymal tumor of the pelvis: a report of two cases. Oncology Letters 20148 67-71. (https:// doi.org/10.3892/ol.2014.2081)

Received in final form 19 April 2018

Accepted 27 April 2018 